\title{
Warburg micro syndrome type 1 associated with peripheral neuropathy and cardiomyopathy
}

\author{
Dagmara Kabzińska ${ }^{1}$, Hanna Mierzewska², Jan Senderek ${ }^{3}$, Andrzej Kochański ${ }^{1}$ \\ ${ }^{1}$ Neuromuscular Unit, Mossakowski Medical Research Centre, Polish Academy of Sciences, Warsaw, Poland, ${ }^{2}$ Department of Pediatric \\ and Adolescent Neurology, Institute of Mother and Child, Warsaw, Poland, ${ }^{3}$ Friedrich Baur Institute, Ludwig Maximilians University \\ of Munich, Munich, Germany
}

\begin{abstract}
The Warburg micro syndrome (WARBM) is a genetically heterogeneous syndrome linked to at least 4 loci. At the clinical level, WARBM is characterized by microcephaly, microphthalmia, microcornea, congenital cataracts, corpus callosum hypoplasia, severe mental retardation, and hypogonadism. In some families additional clinical features have been reported. The presence of uncommon clinical features (peripheral neuropathy, cardiomyopathy) may result in misdirected molecular diagnostics. Using the next generation sequencing approach (NGS), we were able to diagnose WARBM 1 syndrome by detection of a new mutation within the RAB3GAP1 gene. We have detected some DNA variants which may be responsible for cardiomyopathy. We did not find any obvious pathogenic mutation within a set of genes known to be responsible for hereditary motor and sensory neuropathy (HMSN). We conclude that: (i) in clinically delineated syndromes, a classical single-gene oriented approach may be not conclusive especially in the presence of rare clinical features, (ii) peripheral neuropathy and cardiomyopathy are rare additional symptoms coexisting with WARBM 1, (iii) a pleiotropic effect of a single point mutation is sufficient to be causative for WARBM1 and (iv) more WARBM-affected patients should be reported to delineate a complete phenotype.
\end{abstract}

Key words: Warburg syndrome, whole-exome sequencing, RAB3GAP1.

\section{Introduction}

The Warburg micro syndrome (WARBM) is a very rare genetic disorder with an unknown frequency reported in a small group of patients (less than 100) around the world.

For the first time in 1993, WARBM was reported in a consanguineous Pakistani marriage in three affected children with mental retardation [18]. In 2005, in turn, WARBM1 was linked to the 2 q21.3 region, in which inactivating mutations within the RAB3GAP1 gene were detected [2]. In recent years, access to molecular genetic analysis has made possible the delineation of the WARBM phenotype. WARBM manifests with microcephaly, microphthalmia, microcornea, congenital cataracts, corpus callosum hypoplasia, hypogonadism and severe mental retardation [6]. Two WARBM-affected sisters were also found to manifest peripheral neuropathy [11].

The vast majority of WARBM patients originate from consanguineous Muslim families [1,2,14]. 
To date, four genes coding for RAB-family proteins have been reported to be mutated in WARBM syndrome, i.e. the RAB3GAP1 gene on chromosome 2q21.3 (MIM\# 602536), RAB3GAP2 (MIM\# 609275) on chromosome $1 \mathrm{q} 41$, the RAB18 gene (MIM\# 602207) located in the 10p12.1 region and finally the TBC1D20 gene (MIM\#611663) linked to the $20 p 13$ locus. At the clinical level, WARBM1-4 subtypes are indistinguishable.

To date, the analysis of WARBM-involved genes has been directed by the presence of typical clinical features. In fact, in previous studies, only a single gene was analyzed and no data from the rest of the genome were available.

A question thus arises as to whether the whole clinical manifestation of WARBM is related to a single mutation in one of the 4 RAB genes.

In the work described here, we performed for the first time a whole-exome sequencing analysis in a Polish six-generational consanguineous WARBM pedigree.

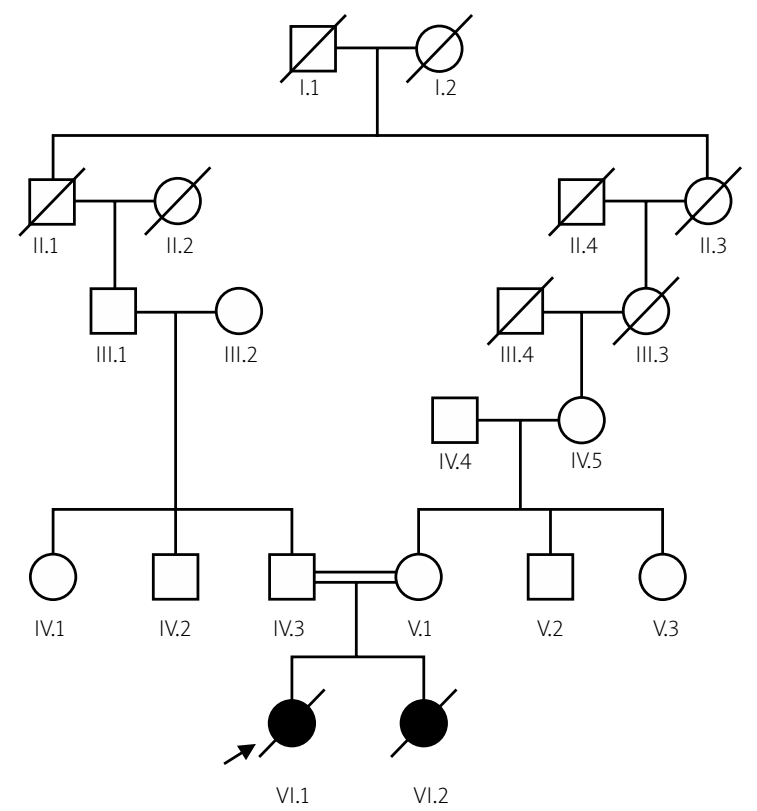

Fig. 1. Pedigree tree of the family. Note the common ancestor within the $1^{\text {st }}$ generation in this consanguineous family tree. The proband is marked with an arrow. Open symbols indicate healthy males (squares) and females (circles). Filled symbols correspond to affected individuals. Deceased individuals are marked with diagonal lines. The double line indicates consanguinity in this family.

\section{Material and methods Case reports}

The patients were two sisters who died in the second decade of life, being the only children of healthy, consanguineous (IV/V) parents (Fig. 1, Fig. 2A-C). They were born following uneventful pregnancies and deliveries. The older girl weighed $2950 \mathrm{~g}$, her body length was $56 \mathrm{~cm}$, OFC was $35 \mathrm{~cm}$, and the Apgar score was 9 points. The younger girl weighed $2850 \mathrm{~g}$, her body length was $54 \mathrm{~cm}$, OFC was $32 \mathrm{~cm}$, and the Apgar score was 10 points. Bilateral congenital cataract was apparent in both girls soon after birth, and a bilateral lentectomy was performed during their infancy.

The girls were severely hypotonic from early life, and their psychomotor development was delayed. At the age of a few months, infantile spasms were observed in the younger sister. Electroencephalography (EEG) showed hypsarrhythmia, so antiepileptic drugs were administered. Electroencephalography of the second sister was also severely abnormal, though no epileptic seizures were observed.

At the age of 2 years they presented with cerebellar ataxia. Limb and axial hypotonia were still observed, but a bilateral Babinski sign was also detected. Mild microcephaly was visible in both girls from early childhood.

Muscle biopsies performed at the ages of 1 and 5 years in the two sisters showed only unspecific changes in muscle fibers with mild lipid accumulation, and without any signs of myopathy or neurogenic changes (data not shown). A biochemical study of OXPHOS revealed slightly decreased activity of complex I, but blood and cerebrospinal fluid lactate were normal, both at baseline and following glucose challenge.

In the second half of the first decade of the patients' lives, mild spasticity developed, but tendon reflexes weakened progressively. Bilateral Babinski signs were still detected, and skeletal anomalies related to chronic muscle hypotonia also developed, in the form of kyphoscoliosis, thoracic deformity, and joint contractures (ulnar, iliofemoral and of the knees). Hands were dropping and planovalgus feet were to be noted. Ophthalmological examination revealed microphthalmia, microcornea and pale, atrophic optic discs. Psychomotor retardation was marked. The patients were never able to sit and walk independently. The younger girl still had epileptic seizures. The EEG of the second sister was also severely abnormal, but seizures were not observed. 

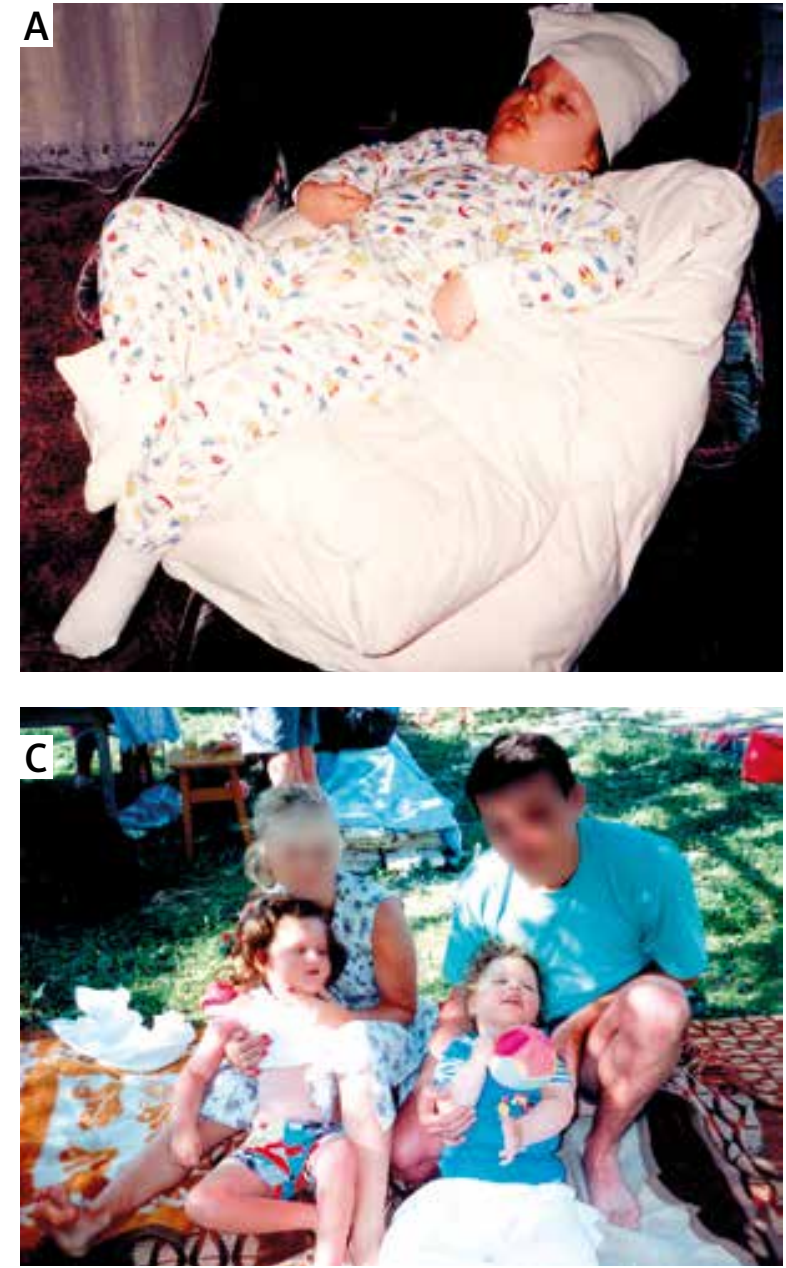

Brain MRI showed slowly progressive cortico-subcortical cerebral and cerebellar atrophy as well as hypomyelination. The corpus callosum was thin and the brainstem was atrophic (Fig. 3). The Nerve Conduction Study (NCS) performed at 7 and 11 years, respectively, revealed progressive motor and sensory neuropathy, with demyelination and mildly expressed axonal degeneration. EMG showed neurogenic changes.

Both girls were of short stature, and deficient as regards body weight. The older girl was hypogonadal. The older girl had cardiomyopathy with left ventricular hypertrophy (148\% of predicted muscle mass). Her arterial blood pressure was normal.

\section{Molecular genetic analysis}

This study was approved by the local Ethical Committee at the Cardinal Stephan Wyszynski University in Warsaw (3/2012 CSWUW). DNA isolated from the proband (VI.1) and her healthy parents (IV.3, V.1) was

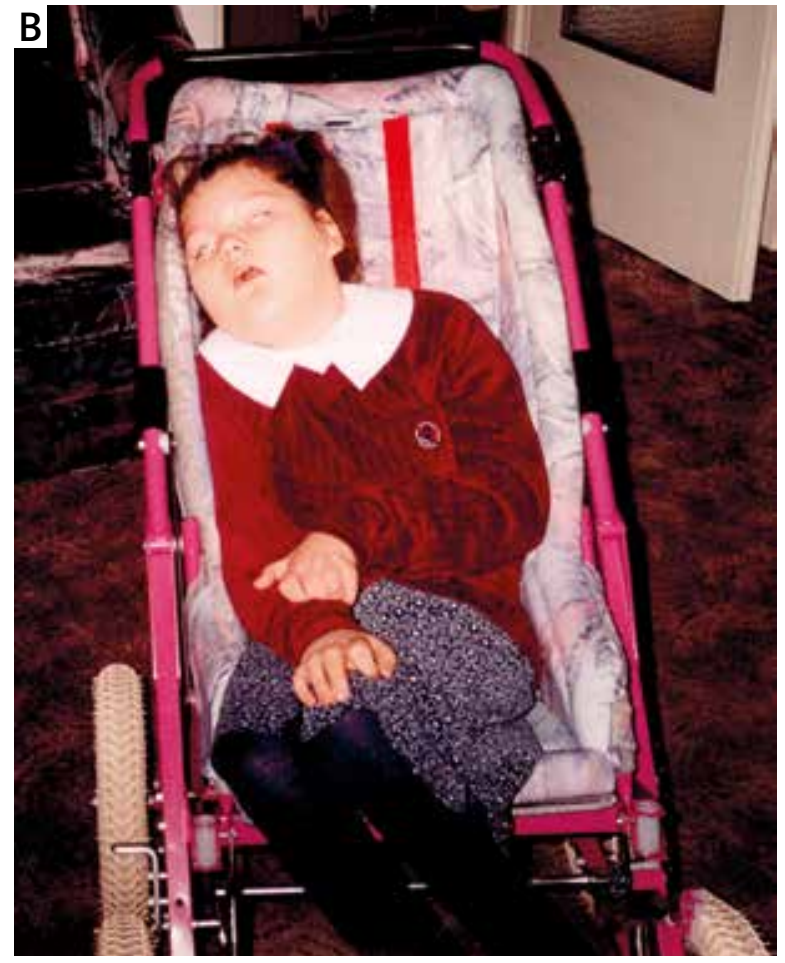

Fig. 2. The patient (J.R.) at the age of $8(\mathbf{A})$ and 10 years (B). Note the dropping hands, thickening of the perioral tissue and anteriorly directed incisor teeth. C) Two sisters at the age of 4 and 8 years. The same types of dysmorphic features are seen. The sisters do not show any similarity to their parents.

isolated from peripheral blood lymphocytes by the salting-out method, following receipt of the patients' informed consent.

\section{PCR and DNA sequencing}

Initially, when we suspected the diagnosis of congenital cataracts, facial dysmorphism and neuropathy (CCFDN), or Marinesco-Sjögren syndrome (MSS), we analysed the CTDP1 and SIL1 genes for mutations in the proband and her healthy parents. The 13 coding exons encompassing the intron-exon boundaries of the CTDP1 gene were amplified using the polymerase chain reaction (PCR) with previously reported primers [19]. The PCR products from the proband and her parents were sequenced directly using a Dynamic terminator cycle sequencing kit (Applied Biosystems) on an ABI PRISM 3700 DNA analyzer (Applied Biosystems). To detect a common CCFDN-causing mutation, i.e. the IVS6+389C >T 

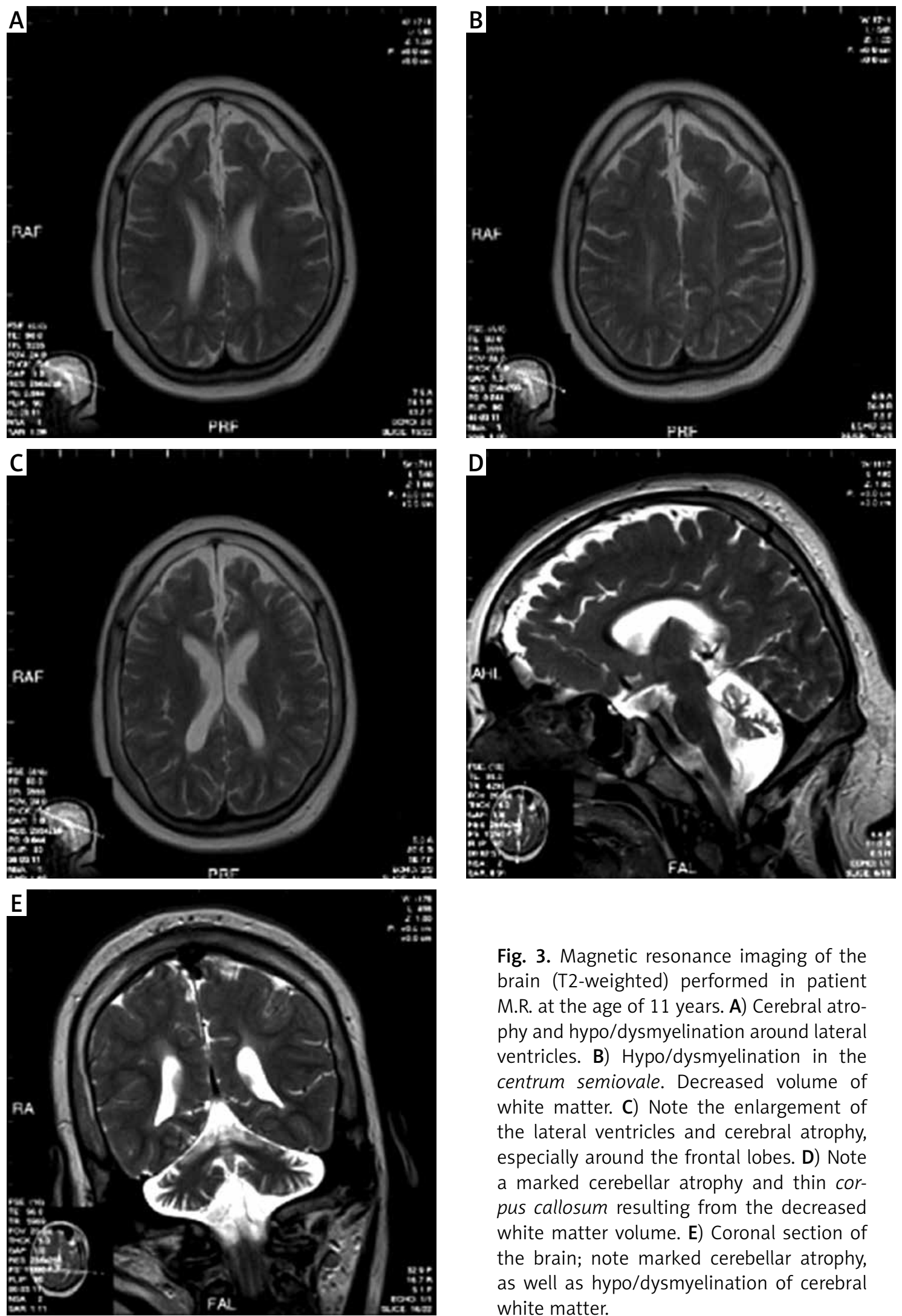

Fig. 3. Magnetic resonance imaging of the brain (T2-weighted) performed in patient M.R. at the age of 11 years. A) Cerebral atrophy and hypo/dysmyelination around lateral ventricles. B) Hypo/dysmyelination in the centrum semiovale. Decreased volume of white matter. C) Note the enlargement of the lateral ventricles and cerebral atrophy, especially around the frontal lobes. D) Note a marked cerebellar atrophy and thin corpus callosum resulting from the decreased white matter volume. E) Coronal section of the brain; note marked cerebellar atrophy, as well as hypo/dysmyelination of cerebral white matter. 
mutation, a restriction analysis with Nla III endonuclease was performed using the forward primer: 5'-CACTGTGTTAGCCAGGATGG-3', and the reverse primer: 5'-GTGCCGTCTGACAGAGATGA-3'. The SIL 1 coding sequence was analysed by direct sequencing of the coding region.

\section{Exome sequencing}

Exome sequencing in the proband (VI.1) and parents (IV.3, V.1) was performed in line with the protocol from Illumina's TruSeq Exome Enrichment Guide. The qualified genomic DNA was fragmented by Covaris, ligated with adapters, purified, amplified and hybridized using a Sure Select Human All Exon $50 \mathrm{Mb}$ Kit (Agilent Technologies), following the manufacturer's instructions. For enrichment, hybridized fragments were bound to the streptavidin beads, and non-hybridised fragments were washed out. The enriched library was then loaded on to a HiSeq 2000 instrument (Illumina). Exome sequencing was performed by Intelliseq sp. z o.o., Cracow.

\section{Exome sequence data analysis}

The sequence reads were analysed using the Illumina pipeline (adapter sequences were removed and low-quality reads discarded). Reads were processed by Picard, and aligned to a human reference sequence (GRCh37) using Bowtie2. BAM files were obtained for each sample using SAM tools, as well as by removing duplicate reads. SNP calling was then performed using GATK. To identify genes with recessive inheritance (the parents are consanguineous [IV/V]), we filtered data by genotype (the proband as a homozygous alternative and the parents as heterozygous, two other control probes being used as a homozygous reference), before dbSNP annotation was carried out and common variants filtered for The SIFT tool was used in the functional and conservation prediction of SNPS.

\section{Mutation confirmation by Sanger sequencing}

The coding sequence with the intron/exon boundaries of the $7^{\text {th }}$ exon of the RAB3GAP1 gene was sequenced in family members by means of Sanger sequencing. Primers (Forward: 5'-CAGTTTGGTATTGTAAGGAGAAA-3' and Reverse: 5'-GCTAACAGACTGAACAAACAA-3') were constructed on the basis of the
NG_01697.1 genomic and NM_001172435.1 RNA sequences.

\section{Analysis of other genes}

To confirm a monogenic basis of the disease in our family we analysed NGS data for all variants in three other genes involved in Warburg micro syndrome diseases, i.e. RAB3GAP2 (NC_000001.11, NM 012414.3),RAB18(NC_000010.11,NM_021252.4)and TBC1D20 (NC_000020.11, NM_144628.3).

Due to the occurrence of cardiomyopathy in one of the affected sisters, additional analysis of variants in 44 genes associated with cardiomyopathy was performed. Additionally, three genes essential in cardiac homeostasis and mitochondrial fusion (MFN1 - mitofusin 1, MFN2 - mitofusin 2 and OPA1 - optic atrophy 1) were analysed.

We established a list of genes on the basis of articles by J.M. Bos et al. 2009, A. Huertas-Vazquea et al. 2013, E. Villard et al. 2011, K. Stark et al. 2010, K.N. Papanicolaou et al. 2012 and Y. Chen et al. 2011 $[4,5,10,15,17,20]$.

\section{Results}

\section{CTDP1 and SIL1 genes}

The IVS6+389C>T mutation in the CTDP1 gene was excluded by means of the RFLP analysis.

The sequencing of the entire CTDP1 coding sequence (including exon-intron boundaries) revealed two sequence variants. In the proband and her mother, a heterozygous C $>\mathrm{T}$ transition at position 1019 resulting (by conceptual translation) in a Thr to Met amino-acid change at codon 340 (T340M) was identified in exon 7 of the CTDP1 gene. The T340M substitution was not detected in the father of the proband. In the proband and her parents, a heterozygous transition $G>A$ at position 2937 in the non-coding region was identified in exon 13 of the CTDP1 gene. Analysis of the SIL 1 gene sequence did not reveal any DNA variant which could be considered a pathogenic mutation for Marinesco-Sjögren syndrome (MSS).

\section{Whole-exome sequencing}

Whole-exome analysis was performed in patient 1 as well as in her healthy mother and father. DNA from patient 2 was not available for the study. After filtering for common variants (>1\%) present in the dbSNP database, we identified 155 variants of poten- 
tial importance. After filtering for nucleotide changes that potentially have a damaging effect on the protein and a high conservation ratio, we obtain only three variants among them, of which only one $-c .538 \mathrm{G}>\mathrm{T}$, p.E180X in the RAB3GAP1 gene - is suitable for the phenotype occurring in patients. Sanger sequencing confirmed the homozygous novel mutation in the proband and a heterozygous one in their healthy parents. The remaining two homozygous variants that were found after filtering were:

- OR13C2 gene (olfactory receptor, family 13 , subfamily C, member 2) rs143198170, NC_0009.12: g. 104605112_104605115delGTTA,NM_001004481.1: c. 513_516delTAAC, p. N171Kfs;

- CNOT1 (CCR4-NOT transcription complex, subunit 1):rs5817153, NC_00016.10:g.58543412delA, NM_016284.4: c. 4434+195delT (isoform a), NM_ 206999.2 (isoform b): c. 4629delT, p. L1544Cfs, NM 001265612.1: c. 4419+195delT (isoform c).

\section{Analysis of other Warburg syndrome genes}

Analysis of other WARBM genes in the proband did not reveal any potentially pathogenic variants. But in the GAP3RAB2 gene a very rare homozygous variant rs2289189 g.220157863C>G (G allele frequency 0.059 ), c.3275G > C with heterozygosity of 0.111 was found, this being described in the dbSNP database as a benign polymorphism.

\section{Analysis of the genes involved in cardiomyopathy}

The analysis of genes associated with cardiomyopathy revealed many SNPs, from among which only rs365990 (g.23392602A>G; c.3302T>C; p.V1101A) in the MYH6 gene had been described previously as associated with a higher risk of cardiac conduction and rhythm disorders $[4,9]$. Moreover, a very rare heterozygous benign polymorphism in the TTN gene called rs55842557 (g.178528964G>A with allele A frequency of only 0.008, c.106787C $>$ T, p.T35596I) was found. We identified that variant in the heterozygous state in the proband and her mother, while no mutation was identified in her father. In the LDB3 gene, a new variant discovered was c.1318 $\mathrm{T}>\mathrm{C}$; p.S440P in VI.1, V.1 (heterozygous) and VI.3 (homozygous), but due to the homozygous variant in the healthy father this mutation probably is not associated with cardiomyopathy occurred in VI.1.
A few missense benign polymorphisms, as well as numerous silent and intron mutations (not shown) were also found in other analysed genes.

\section{Discussion}

Surprisingly, even in the syndromes characterized by a peculiar morphological phenotype, molecular diagnostics may be hampered by the presence of rarely occurring symptoms. The peripheral demyelinating neuropathy and cardiomyopathy are not representative features of WARBM, however peripheral neuropathy was previously reported in two affected sisters harboring mutation within the TBC1D20 gene [11].

Given the presence of peripheral neuropathy in our patients, we started molecular diagnostics from the syndromes in which peripheral neuropathy is a typical clinical feature.

At the beginning of our study we suspected a diagnosis of congenital cataract facial dysmorphism neuropathy (CCFDN), or Marinesco-Sjögren syndrome (MSS). In a classical phenotype-oriented gene analysis we have excluded the mutations within the CTDP1 and SIL1 genes that are respectively causative for CCFDN and MSS.

Due to a lack of conclusive results using the classical Sanger sequencing approach, we decided to perform whole-exome sequencing analysis (WES) in this family.

It is an open question as to whether RAB3GAP1 mutations alone are sufficient to result in a complete phenotype of WARBM1 patients. To the best of our knowledge, the WES analysis was not widely performed to date in WARBM patients. Thus, the potential impact of other genes has not been analyzed so far. Thus, given the large family of RAB proteins and RAB modulating factors, a question arises in regard to the penetrance of the RAB3GAP1 mutation. In fact, the patients detected by us manifested with a complete phenotype of WARBM1. In the exome analysis we found only one deleterious RAB3GAP1 mutation; and no pathogenic mutations have been detected in other RAB and RAB-related genes. Thus, it is highly probable that a single point mutation within the RAB3GAP1 gene is the one and only cause of WARBM 1 disease. This is especially the case given that mutations in the RAB3GAP1 gene associated with WARBM1 were described in families of very varied ethnic origin, with the number of mutations identified already exceeding 50 [14]. The clinical picture of the WARBM1 in our patients 
corresponds with the phenotype described by other authors [7]. However, not typically, in our patients peripheral demyelinating motor-sensory polyneuropathy and cardiomyopathy were identified.

Interestingly, in a series of WARBM individuals with TBC1D20 mutations, only two sisters were found to have demyelinating polyneuropathy [11]. The occurrence of peripheral demyelinating neuropathy within WARBM-affected patients is hard to estimate, since only in some have electromyographic investigations been reported.

In the whole-exome approach used in this study, we did not find any obvious deleterious mutations in the genes involved in the molecular pathogenesis of hereditary motor and sensory neuropathies (HMSN). Thus, due to a lack of mutations within known HMSN genes, we tend to consider peripheral neuropathy rather as a part of the WARBM1 phenotype than an additional clinical feature associated with a mutation of another gene (overlapping syndrome).

Cerebral and cerebellar atrophy, as well as hypoplasia of the corpus callosum seem to be hallmarks of WARBM $[3,6,13]$. In our patients too, it was possible to observe cerebral and cerebellar atrophy with hypoplasia of the corpus callosum. The former sign is a typical finding connected with, and resulting from, hypomyelination. The patients reported by us died in the second decade of life. The nonsense E180X mutation in the RAB3/GAP1 mutation may be at least partially responsible for the poor clinical outcome in these patients.

In one of our patients, cardiomyopathy with left ventricular hypertrophy was detected. Since earlyonset hypertrophic cardiomyopathy is often associated with the mutation of one of the sarcomere genes, we decided to screen the most often mutated genes using an exome approach. We did not detect any pathogenic mutation for the hypertrophic cardiomyopathy. It only proved possible to detect one heterozygous benign polymorphism - rs365990 in the MYH6 gene - this being previously described as associated with a high risk of cardiac conduction and rhythm disorders $[4,8,9]$; as well as the heterozygous rare polymorphism rs55842557 in the TTN gene. Moreover, polymorphismc.1924-851A>G(rs6730157) in the RAB3GAP1 gene has been recently described as being associated with effects on total cholesterol and high density lipoprotein cholesterol levels, and with an increased risk of sudden cardiac death [12].
For this reason, it is not definitively possible to preclude hypertrophic cardiomyopathy belonging to the WARBM syndrome phenotype.

Recently, in the consanguineous family with Kurdish-Armenian descent a large deletion encompassing exons 4-15 of the RAB3GAP1 gene has been found in the siblings with the WARBM1 syndrome. Interestingly, in this pair of patients a mild growth hormone deficiency was detected, and X-ray examination revealed severe osteopenia, which have been not detected to date in WARBM [16]. For molecular diagnostic purposes, the lack or presence of some atypical symptoms should not preclude the molecular analysis of the $R A B$ genes. Moreover, due to a limited number of reported patients, the frequency of WARBM-associated symptoms could not be estimated. Noteworthy, clinical variability of WARBM in various age-groups of patients is also unknown. Thus, a minimal set of symptoms which are necessary for WARBM diagnosis and decision concerning molecular diagnostics may be misleading.

In fact, the number of WARBM reported patients is still too low for reliable phenotype-genotype correlations to be derived.

To summarize, the patients reported by us manifest with a severe clinical form of WARBM1 associated with peripheral demyelinating neuropathy and cardiomyopathy.

Due to the unique structure of the family tree in this study (a recessive trait of inheritance and a detected common ancestor in the $6^{\text {th }}$ generation), we were able to prioritize the filtering process in the interpretation of the exome data. The exome analysis identified only three probably important homozygous sequence variants in the proband. Additionally, only one sequence variant was detected in the $R A B$ $3 G A P 1$ gene whose mutations had been shown previously to segregate with the phenotype of WARBM. Finally, by means of conceptual translation, the identified sequence variant within the RAB3GAP1 gene resulted in a homozygous nonsense E180X mutation truncating the RAB3GAP1 protein. Our study confirms the usefulness of WES analysis in the pedigrees with an evident recessive trait of inheritance and evidence of the common ancestor in the family.

Because of the non-specificity of signs and symptoms of many disorders, molecular genetics is indispensable in establishing a specific diagnosis. In fact, exome analysis seems to be very serviceable in cases of unknown background. Whole-exome sequenc- 
ing in our family gives a chance for the battery of biochemical, imaging and other tests to be omitted. In fact, exome analysis is a cost- and time-effective approach. Most importantly, it also reduces the discomfort of affected patients associated with a long diagnostic process.

\section{Acknowledgements}

This study was supported by the Grant for the Polish National Science Centre No 2012/07/B/NZ4/ 01748 to D.K. We thank Jadwiga Kędzierska for technical support.

\section{Disclosure}

\section{Authors report no conflict of interest.}

\section{References}

1. Ainsworth JR, Morton JE, Good P, Woods CG, George ND, Shield JP, Bradbury J, Henderson MJ, Chhina J. Micro syndrome in Muslim Pakistan children. Ophthalmology 2001; 108: 491-497.

2. Aligianis IA, Johnson CA, Gissen P, Chen D, Hampshire D, Hoffmann K, Maina EN, Morgan NV, Tee L, Morton J, Ainsworth JR, Horn D, Rosser E, Cole TR, Stolte-Dijkstra I, Fieggen K, ClaytonSmith J, Megarbane A, Shield JP, Newbury-Ecob R, Dobyns WB, Graham JM, Jr., Kjaer KW, Warburg M, Bond J, Trembath RC, Harris LW, Takai Y, Mundlos S, Tannahill D, Woods CG, Maher ER. Mutations of the catalytic subunit of RAB3GAP cause Warburg Micro syndrome. Nat Genet 2005; 37: 221-223.

3. Bem D, Yoshimura S, Nunes-Bastos R, Bond FC, Kurian MA, Rahman F, Handley MT, Hadzhiev Y, Masood I, Straatman-Iwanowska AA, Cullinane AR, McNeill A, Pasha SS, Kirby GA, Foster K, Ahmed Z, Morton JE, Williams D, Graham JM, Dobyns WB, Burglen L, Ainsworth JR, Gissen P, Muller F, Maher ER, Barr FA, Aligianis IA. Loss-of-function mutations in RAB18 cause Warburg micro syndrome. Am J Hum Genet 2011; 88: 499-507.

4. Bos JM, Towbin JA, Ackerman MJ. Diagnostic, prognostic, and therapeutic implications of genetic testing for hypertrophic cardiomyopathy. J Am Coll Cardiol 2009; 54: 201-211.

5. Chen Y, Liu Y, Dorn GW. Mitochondrial fusion is essential for organelle function and cardiac homeostasis. Circ Res 2011; 109: 1327-1331.

6. Derbent M, Agras PI, Gedik S, Oto S, Alehan F, Saatci U. Congenital cataract, microphthalmia, hypoplasia of corpus callosum and hypogenitalism: report and review of Micro syndrome. Am J Med Genet A 2004; 128A: 232-234.

7. Handley MT, Morris-Rosendahl DJ, Brown S, Macdonald F, Hardy C, Bem D, Carpanini SM, Borck G, Martorell L, Izzi C, Faravelli F, Accorsi P, Pinelli L, Basel-Vanagaite L, Peretz G, AbdelSalam GM, Zaki MS, Jansen A, Mowat D, Glass I, Stewart H, Mancini G, Lederer D, Roscioli T, Giuliano F, Plomp AS, Rolfs A, Graham JM, Seemanova E, Poo P, Garcia-Cazorla A, Edery P, Jackson IJ, Maher ER, Aligianis IA. Mutation spectrum in RAB3GAP1, RAB3GAP2, and RAB18 and genotype-phenotype cor- relations in warburg micro syndrome and Martsolf syndrome. Hum Mutat 2013; 34: 686-696.

8. Holm H, Gudbjartsson DF, Arnar DO, Thorleifsson G, Thorgeirsson G, Stefansdottir H, Gudjonsson SA, Jonasdottir A, Mathiesen EB, Njolstad I, Nyrnes A, Wilsgaard T, Hald EM, Hveem K, Stoltenberg C, Lochen ML, Kong A, Thorsteinsdottir U, Stefansson K. Several common variants modulate heart rate, PR interval and QRS duration. Nat Genet 2010; 42: 117-122.

9. Holm H, Gudbjartsson DF, Sulem P, Masson G, Helgadottir HT, Zanon C, Magnusson OT, Helgason A, Saemundsdottir J, Gylfason A, Stefansdottir H, Gretarsdottir S, Matthiasson SE, Thorgeirsson GM, Jonasdottir A, Sigurdsson A, Stefansson H, Werge T, Rafnar T, Kiemeney LA, Parvez B, Muhammad R, Roden DM, Darbar D, Thorleifsson G, Walters GB, Kong A, Thorsteinsdottir U, Arnar DO, Stefansson K. A rare variant in MYH6 is associated with high risk of sick sinus syndrome. Nat Genet 2011; 43: 316-320.

10. Huertas-Vazquez A, Nelson CP, Guo X, Reinier K, Uy-Evanado A, Teodorescu C, Ayala J, Jerger K, Chugh H, Braund PS, Deloukas P, Hall AS, Balmforth AJ, Jones M, Taylor KD, Pulit SL, Newton-Cheh C, Gunson K, Jui J, Rotter JI, Albert CM, Samani NJ, Chugh SS. Novel loci associated with increased risk of sudden cardiac death in the context of coronary artery disease. PLoS One 2013; 8: e59905.

11. Liegel RP, Handley MT, Ronchetti A, Brown S, Langemeyer L, Linford A, Chang B, Morris-Rosendahl DJ, Carpanini S, Posmyk R, Harthill V, Sheridan E, Abdel-Salam GM, Terhal PA, Faravelli F, Accorsi P, Giordano L, Pinelli L, Hartmann B, Ebert AD, Barr FA, Aligianis IA, Sidjanin DJ. Loss-of-function mutations in TBC1D20 cause cataracts and male infertility in blind sterile mice and Warburg micro syndrome in humans. Am J Hum Genet 2013; 93:1001-1014.

12. Ma L, Yang J, Runesha HB, Tanaka T, Ferrucci L, Bandinelli S, Da Y. Genome-wide association analysis of total cholesterol and high-density lipoprotein cholesterol levels using the Framingham heart study data. BMC Med Genet 2010; 11: 55.

13. Megarbane A, Choueiri R, Bleik J, Mezzina M, Caillaud C. Microcephaly, microphthalmia, congenital cataract, optic atrophy, short stature, hypotonia, severe psychomotor retardation, and cerebral malformations: a second family with micro syndrome or a new syndrome? J Med Genet 1999; 36: 637-640.

14. Morris-Rosendahl DJ, Segel R, Born AP, Conrad C, Loeys B, Brooks SS, Muller L, Zeschnigk C, Botti C, Rabinowitz R, Uyanik G, Crocq MA, Kraus U, Degen I, Faes F. New RAB3GAP1 mutations in patients with Warburg Micro Syndrome from different ethnic backgrounds and a possible founder effect in the Danish. Eur J Hum Genet 2010; 18: 1100-1106.

15. Papanicolaou KN, Kikuchi R, Ngoh GA, Coughlan KA, Dominguez I, Stanley WC, Walsh K. Mitofusins 1 and 2 are essential for postnatal metabolic remodeling in heart. Circ Res 2012; 111: 1012-1026.

16. Picker-Minh S, Busche A, Hartmann B, Spors B, Klopocki E, Hübner C, Horn D, Kaindl AM. Large homozygous RAB3GAP1 gene microdeletion causes Warburg Micro Syndrome 1. Orphanet J Rare Dis 2014; 9: 113.

17. Stark K, Esslinger UB, Reinhard W, Petrov G, Winkler T, Komajda M, Isnard R, Charron P, Villard E, Cambien F, Tiret L, Aumont MC, Dubourg O, Trochu JN, Fauchier L, DeGroote P, 
Richter A, Maisch B, Wichter T, Zollbrecht C, Grassl M, Schunkert H, Linsel-Nitschke P, Erdmann J, Baumert J, Illig T, Klopp N, Wichmann HE, Meisinger C, Koenig W, Lichtner P, Meitinger T, Schillert A, Konig IR, Hetzer R, Heid IM, Regitz-Zagrosek V, Hengstenberg C. Genetic association study identifies HSPB7 as a risk gene for idiopathic dilated cardiomyopathy. PLoS Genet 2010; 6: e1001167.

18. Warburg M, Sjo O, Fledelius HC, Pedersen SA. Autosomal recessive microcephaly, microcornea, congenital cataract, mental retardation, optic atrophy, and hypogenitalism. Micro syndrome. Am J Dis Child 1993; 147: 1309-1312.

19. Varon R, Gooding R, Steglich C, Marns L, Tang H, Angelicheva D, Yong KK, Ambrugger P, Reinhold A, Morar B, Baas F, Kwa M, Tournev I, Guerguelcheva V, Kremensky I, Lochmuller H, Mullner-Eidenbock A, Merlini L, Neumann L, Burger J, Walter M, Swoboda K, Thomas PK, von Moers A, Risch N, Kalaydjieva L. Partial deficiency of the C-terminal-domain phosphatase of RNA polymerase II is associated with congenital cataracts facial dysmorphism neuropathy syndrome. Nat Genet 2003; 35: 185-189.

20. Villard E, Perret C, Gary F, Proust C, Dilanian G, Hengstenberg C, Ruppert V, Arbustini E, Wichter T, Germain M, Dubourg O, Tavazzi L, Aumont MC, DeGroote P, Fauchier L, Trochu JN, Gibelin P, Aupetit JF, Stark K, Erdmann J, Hetzer R, Roberts AM, Barton PJ, Regitz-Zagrosek V, Aslam U, Duboscq-Bidot L, Meyborg M, Maisch B, Madeira H, Waldenstrom A, Galve E, Cleland JG, Dorent R, Roizes G, Zeller T, Blankenberg S, Goodall AH, Cook S, Tregouet DA, Tiret L, Isnard R, Komajda M, Charron P, Cambien F. A genome-wide association study identifies two loci associated with heart failure due to dilated cardiomyopathy. Eur Heart J 2011; 32: 1065-1076. 